\title{
EIGENVALUE CHARACTERIZATION AND COMPUTATION FOR THE LAPLACIAN ON GENERAL 2-D DOMAINS
}

\author{
Patrick Guidotti $^{1}$ and James V. Lambers ${ }^{2}$ \\ ${ }^{1}$ Department of Mathematics, University of California at Irvine, \\ Irvine, California, USA \\ ${ }^{2}$ Department of Energy Resources Engineering, Stanford University, \\ Stanford, California, USA
}

\begin{abstract}
$\square \quad$ In this paper, we address the problem of determining and efficiently computing an approximation to the eigenvalues of the negative Laplacian $-\triangle$ on a general domain $\Omega \subset \mathbb{R}^{2}$ subject to homogeneous Dirichlet or Neumann boundary conditions. The basic idea is to look for eigenfunctions as the superposition of generalized eigenfunctions of the corresponding free space operator, in the spirit of the classical method of particular solutions (MPS). The main novelties of the proposed approach are the possibility of targeting each eigenvalue independently without the need for extensive scanning of the positive real axis and the use of small matrices. This is made possible by iterative inclusion of more basis functions in the expansions and a projection idea that transforms the minimization problem associated with MPS and its variants into a relatively simple zero-finding problem, even for expansions with very few basis functions.
\end{abstract}

Keywords Dirichlet; Inverse spectral problem; Laplacian eigenvalue problem; Neumann.

AMS Subject Classification 35P15; 65N25.

\section{INTRODUCTION}

The numerical computation of eigenvalues of differential operators is usually performed following two main philosophies. On the one hand, one starts out with a discretization of the differential operator by a finite dimensional matrix approximation obtained by finite differences, finite elements, or any other numerical method. The spectrum of the approximation is then taken as an approximation to the spectrum of the original differential operator. This procedure has two main disadvantages.

Address correspondence to James V. Lambers, Department of Energy Resources, Stanford University, Stanford, CA 94305-2220, USA; E-mail: lambers@stanford.edu 
First, it introduces a number of spurious modes that have nothing to do with the differential operator. Second, the size of the discretization matrix grows with the number of eigenvalues to be computed. For this type of approach we refer to [1, 2] for algorithms using finite elements and finite differences, respectively. One advantage of this method is of course that it can be applied to a wide class of operators, not necessarily with constant coefficients. The other, known as the method of particular solutions (MPS), uses special function series and seeks the singular values of matrices obtained by imposing boundary conditions to their discretization. It was first introduced in [3] and has recently been revived by [4], where the authors resolve a major shortcoming of the method that had been already observed in the original [3]. In its current form as formulated by [4], we shall refer to it as the modified MPS method.

Other more specialized methods can also be found in the literature [2], but they are restricted to special cases where the domain has very high symmetry properties. As for the characterization of eigenvalues, the undoubtedly most used one is that by the Rayleigh quotient, which can equally be applied to continuous as well as discrete approximations.

The method presented here is similar in spirit to the original MPS and exhibits similar difficulties. They are partly overcome by a novel algorithm that is characterized by the use of a secant search, a successive increase of terms in the basis expansion, and a projection idea that significantly improves the deteriorating condition observed as more terms in the expansion are included. The final product is a method that is more accurate than MPS but not as accurate as the modified MPS (for domains with singular corners) but does not require the introduction of interior points or extensive scanning of the real line in the search for eigenvalues. It can therefore be applied in conjunction with the modified MPS by providing a good initial guess and deliver a method that is better than either used independently.

A major problem encountered in the application of MPS is the extremely bad conditioning of the matrices obtained by enforcing the boundary condition on the expansion. Eigenvalues correspond with dips in the smallest singular values of such matrices. As the number of basis functions is increased, the width of these dips narrows very rapidly (Fig. 1), making it very hard to locate the minimal singular values.

The methodology proposed here mitigates this phenomenon by gradually increasing the number of basis functions and by the use of a projection method that allows "preconditioning" of the matrix and effectively leads to wells that are wide enough to make a fast derivative-free minimization practical (see Fig. 9). We also observe that, unlike the modified MPS method, ours allows for a trivial computation of approximate eigenfunctions on the basis of Fourier-Bessel functions once a singular value is found, just as in the original MPS, whereas in the 
modified MPS, as mentioned in [4], solution of an ill-conditioned system is required to obtain these coefficients.

Our method works best for smooth domains but still delivers an improvement over MPS even for "hostile" domains with singular corners. The methodology described above can, however, be applied to more appropriate expansions in terms of eigenfunctions for the Laplacian on sectors to better capture the singular behavior in the corners. In this case, it is not yet clear what the best choice of projection should be.

The proposed method remains stable across a wide range of domains and does not need domain-specific information. The latter quality, combined with its efficiency, makes the method particularly appealing for use in the resolution of inverse spectral problems. A simple experiment in this direction is presented in Subsection 4.5.

We also mention our motivation for the study of this problem. We envision the use of the computed eigenfunctions as trial functions for Galerkin methods for partial differential equations (PDEs). In particular, such trial functions are the best choices for Krylov subspace spectral methods (see [6]) applied to time-dependent problems.

The rest of the paper is organized as follows. In the next section, the problem is formulated and the central ansatz is introduced. The algorithm is described in Section 3, and a number of numerical experiments and comparisons are performed in Section 4.

\section{PRELIMINARIES}

Consider the eigenvalue problem

$$
\begin{cases}-\Delta \varphi=\lambda \varphi & \text { in } \Omega \\ \varphi=0 & \text { on } \partial \Omega\end{cases}
$$

The approach proposed here stems from the natural generalization of the one-dimensional ansatz for the eigenfunctions and reads in this case

$$
\varphi=\int_{\mathbb{S}_{r}^{n-1}} e^{i x \cdot \xi} f(\xi) d \sigma_{\mathbb{S}_{r}^{n-1}}(\xi)=\left\langle f, e^{i x \cdot \xi}\right\rangle_{\mathbb{S}_{r}^{n-1}}
$$

where $\mathbb{S}_{r}^{n-1}$ denotes the sphere of radius $r>0$ in $\mathbb{R}^{n}$. For any choice of a density function (which can happen to be a generalized function), (2.2) is an eigenfunction of the negative Laplacian to the eigenvalue $r^{2}$. An eigenvalue of (2.1) is obtained whenever

$$
\int_{\mathbb{S}_{r}^{n-1}} e^{i x \cdot \xi} f(\xi) d \sigma_{\mathbb{S}_{r}^{n-1}}(\xi)=0, \quad x \in \partial \Omega,
$$


possesses a nontrivial solution $f$. Even though this procedure is dimension independent, we now specialize to the case $n=2$ because the suitable special functions change with dimension. Expanding the density $f$ in a Fourier series

$$
f(\theta)=\sum_{m \in \mathbb{Z}} \alpha_{m} e^{i m \theta}, \quad \theta \in[0,2 \pi),
$$

and substituting in (2.3), the problem can be recast as

$$
\sum_{m \in \mathbb{Z}} \alpha_{m} J_{m}(r|x|) e^{i m \theta_{x}}=0, \quad x \in \partial \Omega,
$$

where $\theta_{x}=\arg \left(x_{1}+i x_{2}\right)$. This follows from Lemma 2.2 below.

Remark 2.1. From this representation, one clearly readily recovers the eigenvalues for the circle as the zeros $\left(r_{k}^{m}\right)_{m, k \in \mathbb{N}}$ of $J_{m}(m \in \mathbb{N})$ and the associated eigenfunctions $\left(J_{m}\left(r_{k}^{m}|x|\right) e^{i m \theta_{x}}\right)_{m, k \in \mathbb{N}}$ corresponding with the nontrivial solutions

$$
\alpha=e_{m}=(0, \ldots, 0, \underbrace{1}_{m \text { th entry }}, 0, \ldots), \quad m \in \mathbb{N},
$$

of (2.5). Observe that $J_{-m}=(-1)^{m} J_{m}$.

This remark is going to play a crucial role in the design of a stable, efficient algorithm for the computation of the eigenvalues and of the Fourier coefficients for the density function in the case of arbitrary domains for which a homotopy to the circle is used in the process. Other boundary conditions can also be considered. We refer the reader to Subsection 3.3.

Lemma 2.2. For any $r>0$, one has that

$$
\frac{1}{2 \pi i^{m}} \int_{0}^{2 \pi} e^{i r x \cdot \xi_{\theta}} e^{i m \theta} d \theta=J_{m}(r|x|) e^{i m \theta_{x}}, \quad x \in \mathbb{R}^{2} .
$$

Hereby $\xi_{\theta}=(\cos \theta, \sin \theta)$. We also make use the standard notation $J_{m}$ for the Bessel functions of integer order.

Proof. First write

$$
x=|x|\left(\cos \theta_{x}, \sin \theta_{x}\right), \quad \theta_{x}=\arg \left(x_{1}+i x_{2}\right)
$$

and observe that

$$
e^{i r x \cdot \xi_{\theta}}=e^{i r|x|\left(\cos \theta_{x} \cos \theta+\sin \theta_{x} \sin \theta\right)}=e^{i r|x| \cos \left(\theta-\theta_{x}\right)}
$$


Then, by virtue of the periodicity of the integrand, it follows that

$$
\int_{0}^{2 \pi} e^{i r x \cdot \xi_{\theta}} e^{i m \theta} d \theta=\int_{0}^{2 \pi} e^{i r|x| \cos \left(\theta-\theta_{x}\right)} e^{i m \theta} d \theta=e^{i m \theta_{x}} \int_{0}^{2 \pi} e^{i r|x| \cos \theta} e^{i m \theta} d \theta .
$$

Finally, it is known that (cf. [8])

$$
\frac{1}{2 \pi i^{m}} \int_{0}^{2 \pi} e^{i r|x| \cos \theta} e^{i m \theta} d \theta=J_{m}(r|x|) .
$$

from which the claim follows.

Example 2.3. To show that the density function $f$ in (2.2) can indeed be a distribution, we consider the unite square centered in the origin. It is elementary to verify that

$$
f=\frac{1}{4} \sum_{j=0}^{3} \delta_{\xi_{j}}, \xi_{j}=(\cos (\pi / 4+j \pi / 2), \sin (\pi / 4+j \pi / 2))
$$

is the density for the first eigenfunction and that any other eigenfunction also has generalized kernel.

Finally, we consider a class of domains that we shall revisit in the numerical experiments to be presented later.

Example 2.4. Let $\partial \Omega$ be a star-shaped domain described by

$$
\rho(\theta)(\cos (\theta), \sin (\theta)), \quad \theta \in[0,2 \pi)
$$

for a given (continuous) periodic function $\rho:[0,2 \pi) \rightarrow[0, \infty)$. In this case, the determining equation reads

$$
\sum_{m \in \mathbb{Z}} \alpha_{m} J_{m}(r \rho(\theta)) e^{i m \theta}=0, \quad \theta \in[0,2 \pi)
$$

It seems natural to set up a system of equations for a truncated version of the series appearing in (2.8) and taking an inner product with finitely many basis functions like, e.g., $e^{i k \theta}, \theta \in[0,2 \pi)$.

Notice that a translation in the domain only results in the multiplication of the density function by a nonvanishing function so we can assume without loss of generality that the domain contains the origin, or, in this case, that it can even be star-shaped with respect to the origin.

In the one-dimensional case, there is a natural ordering of the eigenvalues according to their size and, more interestingly, by the number 
of nodal points of the associated eigenfunction. A similarly precise characterization in higher dimension is most likely out of reach except in special cases. We refer to [7] for some results in this direction. There is, however, a way of predicting the rough frequency content of an eigenfunction corresponding with an eigenvalue of a given size. This is related to a property of Bessel functions, according to which $J_{m}(m t)$ is exponentially decaying in $m$ for $0<t<1$ (cf. [8]). In the case of a star-shaped domain, this means that $J_{m}$ becomes exponentially small for $m$ beyond $R\|\rho\|_{\infty}$. This property clearly points to the conditioning problems observed when the number of terms in the expansion is increased.

\section{COMPUTATION}

In this section, we assume $\Omega$ is a star-shaped domain with boundary $\partial \Omega$ described by (2.7). We will describe an iterative method for computing an approximate solution of the determining equation (2.8) for the coefficients $\left\{\alpha_{m}\right\}$ in the Fourier expansion (2.4) of the density $f(\theta)$.

The main idea is to begin with a domain for which we know exact eigenpairs, a circle. The eigenvalues will serve as our initial guesses for the eigenvalues of $-\Delta$ on $\Omega$, and the eigenfunctions serve as trivial expansions of the form (2.5), containing only one term of the form $J_{m_{0}}(\sqrt{\lambda}) e^{i m_{0} \theta}$ for some integer $m_{0}$. Then, our iteration will deform the circle into the shape defined by $\partial \Omega$ and add terms to the eigenfunction expansions until eigenpairs of $\Omega$ are found.

\subsection{Definitions}

Let $M$ and $m_{0}$ be nonnegative integers representing the size and center, respectively, of the Fourier-Bessel expansion of an approximate eigenfunction. We define the set of indices $I\left(M, m_{0}\right)$ by

$$
I\left(M, m_{0}\right)=\left\{-m_{0}-M, \ldots,-m_{0}+M\right\} \cup\left\{m_{0}-M, \ldots, m_{0}+M\right\} .
$$

We denote by $\left|I\left(M, m_{0}\right)\right|$ the number of indices in this set, and we use the notation $I_{j}\left(M, m_{0}\right)$ to refer to the $j$ th index in the set, when arranged in increasing order. These indices correspond with the orders of the Bessel functions that are included in an eigenfunction expansion of the form (2.5).

For convenience, we define

$$
\varphi_{m}(r, \theta)=J_{m}(r \rho(\theta)) e^{i m \theta}, \quad m \in I\left(M, m_{0}\right)
$$


We then define an $n$-point grid on the interval $[0,2 \pi)$ with gridpoints $0 \leq \theta_{1}<\theta_{2}<\cdots<\theta_{n}<2 \pi$. Next, we define $\Phi_{n}\left(r, M, m_{0}\right)$ to be an $n \times\left|I\left(M, m_{0}\right)\right|$ matrix with entries

$$
\left[\Phi_{n}\left(r, M, m_{0}\right)\right]_{i j}=\frac{\varphi_{I_{j}\left(M, m_{0}\right)}\left(r, \theta_{i}\right)}{\left(\sum_{k=1}^{n}\left|\varphi_{I_{j}\left(M, m_{0}\right)}\left(r, \theta_{k}\right)\right|^{2}\right)^{1 / 2}} .
$$

It can be seen from the definition that each column of $\Phi_{n}\left(r, M, m_{0}\right)$ has unit 2-norm.

Finally, we let $Q_{n}\left(\epsilon, M, m_{0}\right)$ be the matrix obtained by orthogonalizing the columns of $\Phi_{n}\left(\epsilon, M, m_{0}\right)$, for a parameter $\epsilon<r$. We define the matrix $A\left(r, M, m_{0}\right)$ by

$$
A\left(r, M, m_{0}\right)=\left[Q_{n}\left(\epsilon, M, m_{0}\right)\right]^{H} \Phi_{n}\left(r, M, m_{0}\right) .
$$

The columns of $\Phi_{n}\left(r, M, m_{0}\right)$ correspond with the Fourier-Bessel functions in the series (2.5). We will seek a value of $r$ such that a linear combination of these functions, when projected into the column space of $Q_{n}\left(\epsilon, M, m_{0}\right)$, nearly vanishes on $\partial \Omega$. In practice, we have found that $\epsilon$ should not be larger than $r$, but a choice that is optimal for a variety of test cases has yet to be determined and is left to future work. Note that the matrices $Q_{n}$ and $\Phi_{n}$ are evaluated with different first arguments, so their column spaces are not the same.

In Section 4.6, we will discuss in greater detail this approach of projecting the columns of $\Phi_{n}\left(r, M, m_{0}\right)$. However, we note here that when the boundary $\rho(\theta)$ is reasonably smooth, then very accurate results can be obtained by simply setting the columns of $Q_{n}\left(r, M, m_{0}\right)$ to be the discretizations of functions of the form $e^{i m \theta}$, for $m \in I\left(M, m_{0}\right)$. In fact, this choice is used to obtain the numerical results in Sections 5.1-5.3.

\subsection{Algorithm Description}

For each eigenvalue $\lambda_{j}$, we seek an approximate eigenfunction $u_{j}(\rho, \theta)$ of the form

$$
u_{j}(\rho, \theta)=\sum_{m \in I\left(M, m_{0}\right)} \alpha_{m} J_{m}\left(\sqrt{\lambda_{j}} \rho\right) e^{i m \theta} .
$$

The coefficients $\left\{\alpha_{m}\right\}_{m \in I\left(M, m_{0}\right)}$ and eigenvalue $\lambda_{j}$ must be chosen so that $u_{j}$ vanishes, at least approximately, on $\partial \Omega$. To compute these values, for given $M$ and $m_{0}$, we can solve the nested minimization problem

$$
\min _{r>0} \min _{\|\mathbf{a}\|_{2}=1}\left\|A\left(r, M, m_{0}\right) \mathbf{a}\right\|_{2}
$$


The vector a represents the coefficients $\alpha_{m}$, for $m \in I_{m}\left(M, m_{0}\right)$, in the expansion (3.2), and $r$ corresponds with the square root of the eigenvalue $\lambda_{j}$.

First, we examine the inner minimization problem for fixed values of $r, M$, and $m_{0}$. We can solve this problem by computing the singular value decomposition, or SVD (see [9, Ch. 8]), of $A\left(r, M, m_{0}\right)$,

$$
A\left(r, M, m_{0}\right)=U \Sigma V^{H},
$$

where $U$ and $V$ are unitary and $\Sigma$ is diagonal with diagonal entries

$$
0 \leq \sigma_{1} \leq \sigma_{2} \leq \cdots \leq \sigma_{\left|I\left(M, m_{0}\right)\right|}
$$

Then, $\left\|A\left(r, M, m_{0}\right) \mathbf{a}\right\|_{2}$ is minimized by setting $\mathbf{a}=\mathbf{v}_{1}$, where $\mathbf{v}_{i}$ is the $i$ th column of $V$ for $i=1,2, \ldots,\left|I\left(M, m_{0}\right)\right|$. It follows that $\left\|A\left(r, M, m_{0}\right) \mathbf{a}\right\|_{2}=\sigma_{1}$.

Observe that it is not necessary to compute the entire SVD to obtain an approximation to $\mathbf{v}_{1}$; one can instead apply, for example, a restarted block Lanczos bidiagonalization method such as those described in [10], which are specifically designed to compute a small number of extremal singular values and associated left and right singular vectors.

Let $\sigma^{*}\left(r, M, m_{0}\right)$ denote the smallest singular value of $A\left(r, M, m_{0}\right)$, which is also the solution of the inner minimization problem. If we can solve the outer minimization problem, seeking an $r$ that minimizes $\sigma^{*}\left(r, M, m_{0}\right)$, and if the solution $r^{*}$ is such that $\sigma^{*}\left(r^{*}, M, m_{0}\right)$ is nearly zero, then the coefficients $\left\{\alpha_{m}\right\}_{m \in I\left(M, m_{0}\right)}$ obtained from the corresponding singular vector $\mathbf{v}_{1}\left(A\left(r^{*}, M, m_{0}\right)\right)$ and the value $\lambda_{j}=\left(r^{*}\right)^{2}$, when substituted into (3.2), yield an approximate eigenfunction of $-\Delta$ on $\Omega$ that satisfies Dirichlet boundary conditions.

We first describe how we choose our initial iterates. We use the eigenvalues for a circle of radius $\rho_{0}$, where

$$
\rho_{0}=\operatorname{Avg}_{\theta \in[0,2 \pi]} \rho(\theta) \text {. }
$$

These eigenvalues are simply the values of $\lambda$ for which $J_{m}\left(\sqrt{\lambda} \rho_{0}\right)=0$, for some nonnegative integer $m$. For $m=0$, the eigenvalues are simple; otherwise they have multiplicity 2 , in which case they are included twice in the set of initial iterates and perturbed slightly, so that closely clustered eigenvalues can be found independently.

For each initial iterate $r$, where $r^{2}$ is an eigenvalue for the circle of radius $\rho_{0}$, we let $m_{0}$ be the integer such that $J_{m_{0}}\left(r \rho_{0}\right)=0$, prior to any perturbation. Then, we begin an iteration to find an eigenvalue. For $M=0,1,2, \ldots$, we minimize

$$
f_{M}(r)=\sigma_{1}\left(A\left(r, M, m_{0}\right)\right)
$$


using the minimizer $r^{*}$ of $f_{M-1}(r)$ as the initial iterate when $M>0$, and the value described in the previous paragraph as the initial iterate when $M=0$. When $M=0$, the expansion (3.2) only includes Bessel functions of order $m_{0}$ and $-m_{0}$. When $M=1$, we add Bessel functions of order $m_{0} \pm 1$ and $-m_{0} \pm 1$; when $M=2$, we add orders $m_{0} \pm 2$ and $-m_{0} \pm 2$, and so on. The set $I\left(M, m_{0}\right)$ describes all orders that are included in the expansion for any choice of $M$ and $m_{0}$.

To minimize $f_{M}(r)$, we exploit the fact that near the minimizer, $f_{M}(r)$ is a continuous, approximately piecewise linear function of $r$, with the minimizer occurring at the boundary between two pieces. Therefore, we can use any derivative-free optimization method to quickly obtain an approximation to the minimizer. However, in using any such method, one should exploit this behavior of $f_{M}(r)$.

To that end, we perform an inner iteration in which we compute each iterate $r_{k}$ by constructing a secant line using the previous iterate $r_{k-1}$ and a small perturbation $r_{k-1}+\delta_{k}$. This perturbation is chosen by assuming that $f_{M}(r)$ is exactly piecewise linear and then choosing the sign of $\delta_{k}$ to ensure that $r_{k-1}+\delta_{k}$ lies on the same piece as $r_{k-1}$. We then obtain $r_{k}$ by computing the $r$-intercept of this secant line.

If $f_{M}\left(r_{k}\right)$ is sufficiently small, then the iteration terminates successfully. Otherwise, we repeat this process with $r_{k}$. If $r_{k}$ is an outlying value relative to the previous iterates (that is, it lies outside the smallest interval containing the iterates and the sequence of iterates is not monotone, indicating it is outside the "well" we are seeking), then it is rejected and a new iterate is chosen based on the previous iterates and their slopes, but this is not a typical occurrence in practice.

The motivation for this approach is illustrated in Figure 1. Note that for smaller values of $M$, one must only have a reasonable initial guess in order to exploit the approximate piecewise linearity of $f_{M}(r)$ near the minimizer. As $M$ increases, the "well" containing the minimizer begins to "close," thus requiring a much more accurate initial guess. If this requirement is not satisfied, the fact that $f_{m}(r)$ is very smooth away from the minimizer implies that this "well" would be missed entirely by a search algorithm that did not take very small steps.

The reason for the closing of the well is that as $M$ increases, $A\left(r, M, m_{0}\right)$ becomes more ill-conditioned, and $\sigma_{1}\left(A\left(r, M, m_{0}\right)\right)$ becomes smaller regardless of the value of $r$. This troublesome trend is mitigated significantly by normalizing the columns of $\Phi\left(r, M, m_{0}\right)$ and orthogonalizing the columns of $\Phi\left(\epsilon, M, m_{0}\right)$, but for sufficiently large $M$ it can still cause difficulties if $r$ is not already close to an eigenvalue.

If the boundary of $\Omega$ is not very smooth, then it is advisable to construct a homotopy from the circle of radius $\rho_{0}$ to $\Omega$, resulting in a sequence of domains $\Omega_{0}, \Omega_{1}, \ldots, \Omega_{\widetilde{M}}$ where each $\Omega_{k}$ is a star-shaped domain with boundary defined by $\rho_{k}(\theta)=t_{k} \rho(\theta)+\left(1-t_{k}\right) \rho_{0}$ 
$m_{0}=0, M=3$

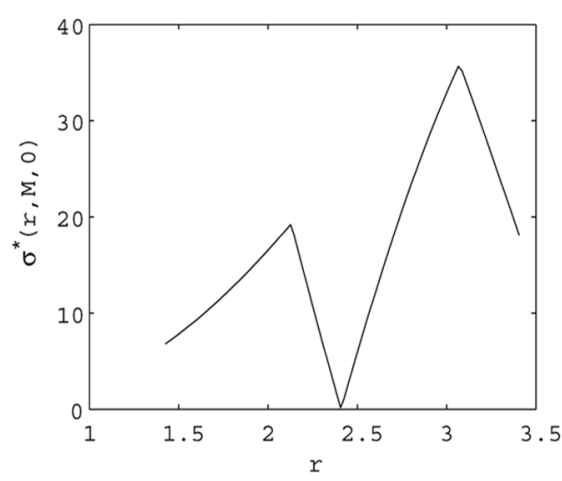

$m_{0}=0, M=5$

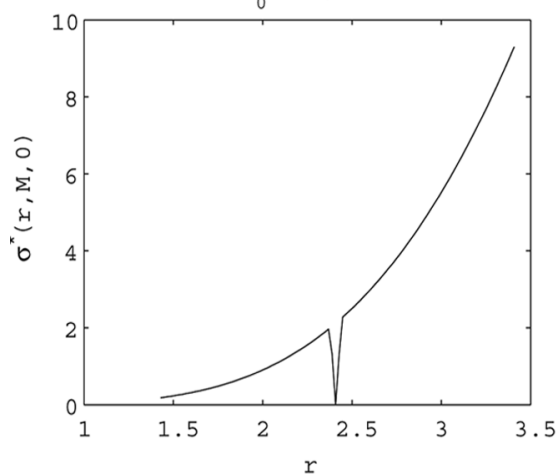

$m_{0}=0, M=4$

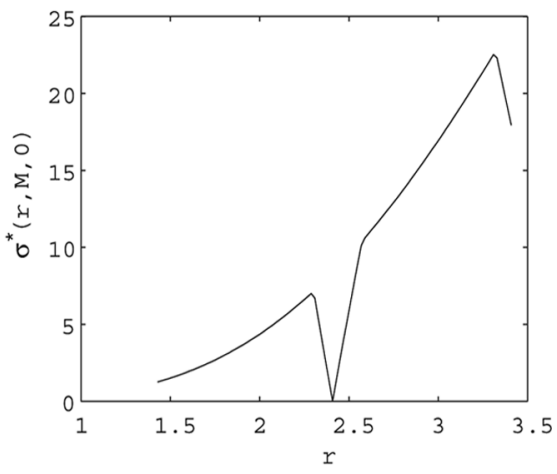

$m_{0}=0, M=6$

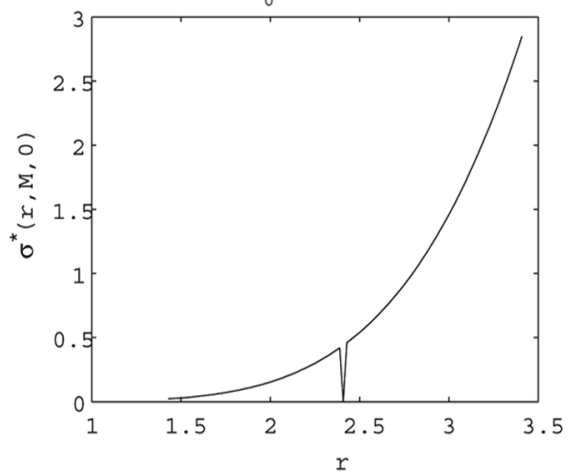

FIGURE 1 Plots of $f_{M}(r)=\sigma^{*}\left(r, M, m_{0}\right)$ for various values of $M$ and $m_{0}=0$. The domain is defined by $\rho(\theta)=1+0.05 \sin (2 \theta)$.

and $0=t_{0}<t_{1}<\cdots<t_{\widetilde{M}-1}<t_{n}=1$. Then, when we are minimizing $f_{M}(r)$ for $M<\tilde{M}$, we are using $\Omega_{M}$ in place of $\Omega$. This homotopy helps to ensure that as $M$ increases, our initial iterate $r_{0}$ is sufficiently close to the minimizer.

Once we have found a minimizer $r^{*}$ of $f_{M}(r)$, we can obtain the approximate solution a of the determining equation (2.8) by computing the right singular vector $\mathbf{v}_{1}$ of the matrix $A\left(r^{*}, M, m_{0}\right)$. This vector $\mathbf{v}_{1}$ represents the coefficients $\alpha_{m}$, for $i \in I_{m}\left(r, M, m_{0}\right)$, in the expansion of the approximate eigenfunction. We then increase the value of $M$ and repeat this process until our approximate eigenvalue $r$ converges to within some tolerance. The discussion at the end of Section 2 suggests an upper limit on $M$.

\subsection{Neumann Boundary Conditions}

With minor modifications, we can find eigenfunctions that satisfy Neumann boundary conditions. For simplicity, we again consider a 
star-shaped domain $\Omega$ defined by the polar equation $r=\rho(\theta)$ for $\theta \in[0,2 \pi)$. For each $\theta$, we define $\phi_{N}(\theta)$ to be the angle that the outward unit normal vector at the boundary point $(\rho(\theta) \cos \theta, \rho(\theta) \sin \theta)$ makes with the positive $x$-axis. Our goal is to solve an equation similar to (2.8),

$$
\sum_{m \in \mathbb{Z}} \alpha_{m} \frac{\partial}{\partial n}\left[J_{m}(r \rho(\theta)) e^{i m \theta}\right]=0, \quad \theta \in[0,2 \pi)
$$

where

$$
\frac{\partial}{\partial n}\left[J_{m}(r \rho(\theta)) e^{i m \theta}\right]=r\left[J_{m-1}(r \rho(\theta)) e^{i\left[(m-1) \theta+\phi_{N}(\theta)\right]}-J_{m+1}(r \rho(\theta)) e^{i\left[(m+1) \theta-\phi_{N}(\theta)\right]}\right] .
$$

This expression was obtained using a well-known recurrence relation for derivatives of Bessel functions, see [11]. We obtain an algorithm analogous to the Dirichlet case, in which we seek $r$ that minimizes $\sigma_{1}\left(A_{N}\left(r, M, m_{0}\right)\right)$, where $M$ and $m_{0}$ are chosen as before and the matrix $A_{N}\left(r, M, m_{0}\right)$ is defined as follows. As in the Dirichlet case, we begin with the set of functions $\left\{\varphi_{m}(r, \theta)\right\}_{m \in I\left(M, m_{0}\right)}$ defined earlier in this section, and a set of gridpoints $\theta_{i=1}^{N}$ chosen from $[0,2 \pi)$.

Using (3.7), we evaluate the normal derivatives of $\varphi_{m}\left(r, \theta_{i}\right)$ for each $m \in I\left(M, m_{0}\right)$ and $i=1, \ldots, n$. These values are stored in a matrix $\Phi_{N}\left(r, M, m_{0}\right)$, in a manner analogous to the definition of $\Phi\left(r, M, m_{0}\right)$ in the Dirichlet case. We then normalize the columns of $\Phi_{N}\left(r, M, m_{0}\right)$ so that each has unit 2-norm, as before. We construct the matrix $\Phi_{N}\left(\epsilon, M, m_{0}\right)$ in the same manner and orthogonalize its columns to obtain $Q_{N}\left(\epsilon, M, m_{0}\right)$. Finally, we obtain

$$
A_{N}\left(r, M, m_{0}\right)=Q_{N}\left(\epsilon, M, m_{0}\right)^{H} \Phi_{N}\left(r, M, m_{0}\right) .
$$

Using this matrix in place of $A\left(r, M, m_{0}\right)$, the algorithm proceeds exactly as in the Dirichlet case, except that if a homotopy from a circle to $\partial \Omega$ is used, the normal vectors for each intermediate boundary must be approximated numerically.

\section{NUMERICAL RESULTS}

We now demonstrate the accuracy of our approach on a number of examples. It will be seen that for "nicer" domains, such as those without singular corners, very high accuracy (close to machine precision) can be achieved. Whereas this is not the case for more difficult domains, we will demonstrate that we can still efficiently obtain reasonably accurate estimates of eigenvalues, using smaller bases than MPS, which, if nothing 
else, can serve as excellent initial guesses for other methods such as the modified MPS of [4] in conjunction with a standard derivative-free optimization method, which would then require very few iterations. That is, the combination of our approach with a method such as MPS can be more effective than either method used alone.

\subsection{Domains with Smooth Boundaries}

First, we consider a star-shaped domain with a smooth boundary, defined by $(2.7)$ with $\rho(\theta)=1+0.05 \sin (2 \theta)$. The domain is shown in Figure 2a. Table 1 lists approximations to the ten smallest eigenvalues. Note that the value of the objective function (which is zero at an eigenvalue, based on the discussion in the preceding sections) is not much larger than machine precision, and that the number of inner iterations is comparable for all eigenvalues. The implementation imposes a limit of at most three inner iterations per outer iteration; that is, we prefer to increase $M$ rather than expend more effort to find an approximate zero of the objective function for a particular value of $M$.

We now examine the coefficients $\alpha_{m}$ of the corresponding eigenfunctions. It can be seen in Figure 3 that for fixed values of $m$, the coefficients $\left\{\tilde{\alpha}_{m}\right\},\left\{\hat{\alpha}_{m}\right\}$ appear to converge rapidly as $M$ increases.

We can also obtain roots for domains whose boundaries exhibit greater oscillation. The domain defined by $(2.7)$ with $\rho(\theta)=1+0.25 \sin (4 \theta)$ is shown in Figure 2b. Table 2 lists approximations to the smallest ten eigenvalues. As can be seen in the table, more iterations and more expansion terms are required than in the previous example.

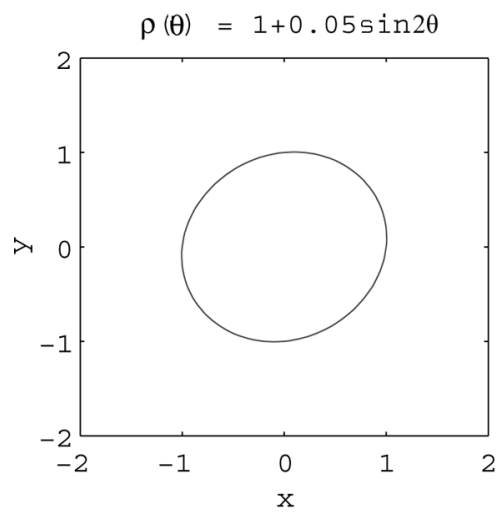

(a)

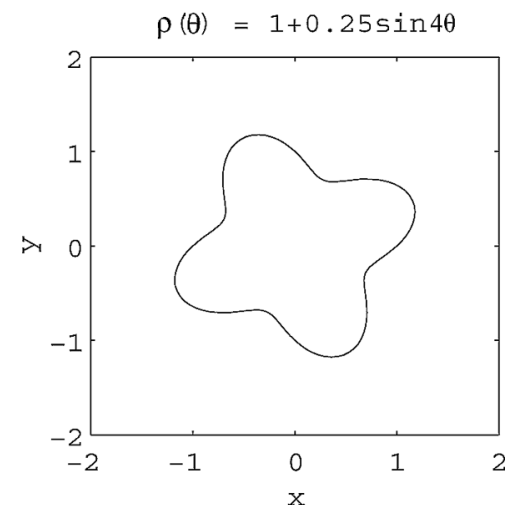

(b)

FIGURE 2 (a) Left plot: domain with boundary described by polar equation $\rho(\theta)=1+0.05 \sin 2 \theta$. (b) Right plot: domain with boundary described by polar equation $\rho(\theta)=1+0.25 \sin 4 \theta$. 
TABLE 1 Eigenvalues of a star-shaped domain with smooth boundary*

\begin{tabular}{rccccc}
\hline$k$ & $m_{0}$ & $M_{\max }$ & $r_{k}=\sqrt{\mu_{k}}$ & $\sigma^{*}\left(r_{k}, M_{\max }, m_{0}\right)$ & Iterations \\
\hline 1 & 0 & 12 & 2.40900956310833 & $5.1915 \mathrm{e}-015$ & 13 \\
2 & 1 & 14 & 3.74095865321159 & $1.4623 \mathrm{e}-015$ & 17 \\
3 & 1 & 14 & 3.93284180810808 & $8.2596 \mathrm{e}-016$ & 17 \\
4 & 2 & 14 & 5.07072160959815 & $1.3518 \mathrm{e}-015$ & 30 \\
5 & 2 & 14 & 5.13847966669082 & $2.843 \mathrm{e}-015$ & 14 \\
6 & 0 & 14 & 5.60117924855279 & $8.3542 \mathrm{e}-015$ & 17 \\
7 & 3 & 14 & 6.35615204542185 & $3.1301 \mathrm{e}-015$ & 18 \\
8 & 1 & 14 & 6.89470061520270 & $5.5334 \mathrm{e}-015$ & 18 \\
9 & 1 & 14 & 7.23051290051418 & $2.4392 \mathrm{e}-015$ & 16 \\
10 & 4 & 14 & 7.55755578029419 & $2.0736 \mathrm{e}-015$ & \\
\hline
\end{tabular}

*Approximations $\left\{\mu_{k}\right\}_{k=1}^{10}$ to the ten smallest eigenvalues $\left\{\lambda_{k}\right\}_{k=1}^{10}$ of $-\Delta$ on the star-shaped domain with boundary given by $\rho(\theta)=1+0.05 \sin (2 \theta), 0 \leq \theta<2 \pi$. The value $M_{\max }$ indicates the largest value of $M$ used. The number of iterations refers to the number of inner iterations.
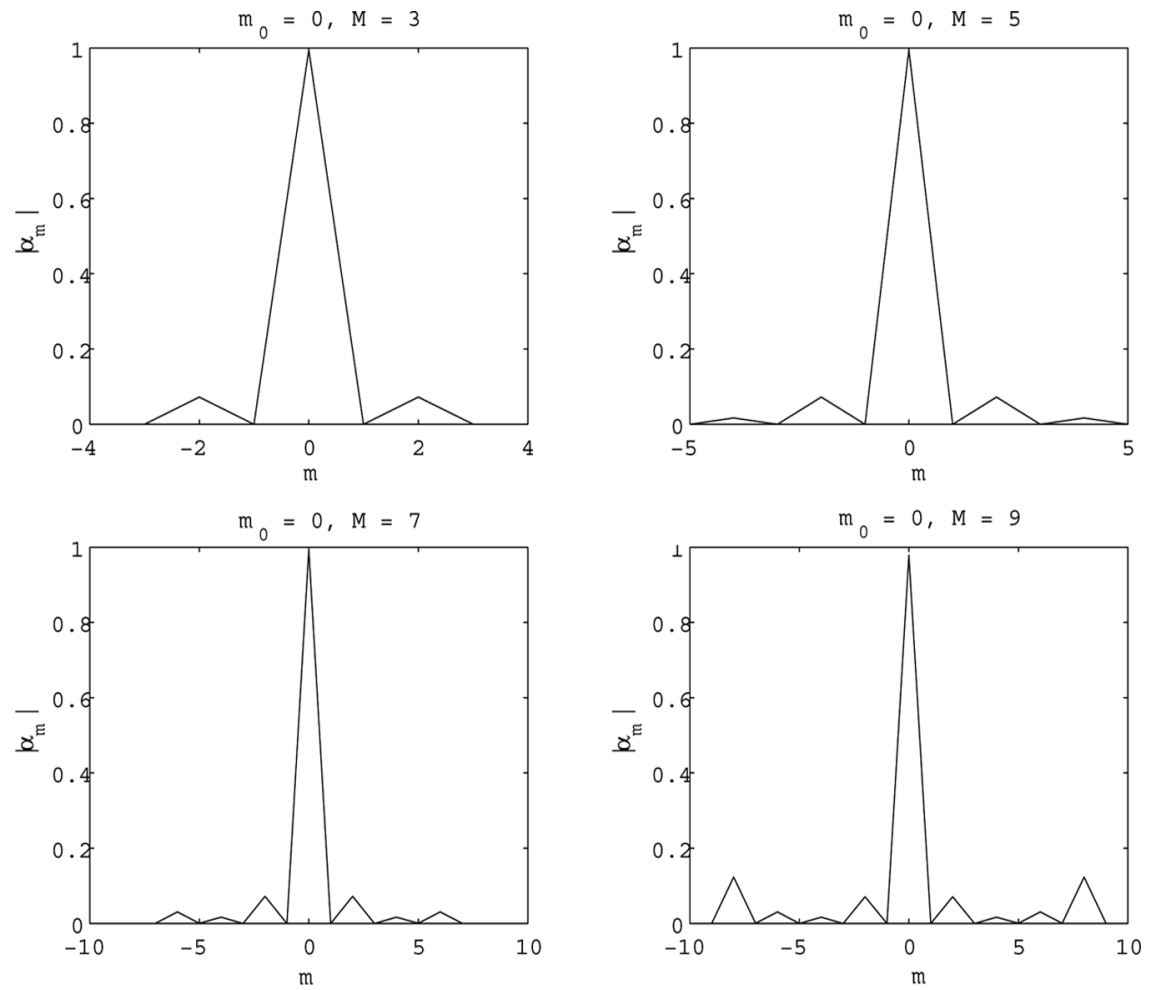

FIGURE 3 For increasing values of $M$, coefficients $\left\{\tilde{\alpha}_{m}\right\}_{m=m_{0}-M}^{m_{0}+M}$ that are approximate solutions of the determining equation (2.8) for eigenfunctions satisfying Dirichlet boundary conditions on the star-shaped domain defined by $\rho(\theta)=1+0.05 \sin (2 \theta)$. 
TABLE 2 Eigenvalues of a star-shaped domain with oscillatory boundary*

\begin{tabular}{rccccc}
\hline$k$ & $m_{0}$ & $M_{\max }$ & $r_{k}=\sqrt{\mu_{k}}$ & $\sigma^{*}\left(r_{k}, M_{\max }, m_{0}\right)$ & Iterations \\
\hline 1 & 0 & 25 & 2.68969299056912 & $2.0328 \mathrm{e}-015$ & 30 \\
2 & 1 & 25 & 4.12955410262284 & $8.1337 \mathrm{e}-016$ & 41 \\
3 & 2 & 25 & 4.85603553243775 & $1.6213 \mathrm{e}-015$ & 29 \\
4 & 2 & 22 & 5.48806069658009 & $2.3608 \mathrm{e}-015$ & 20 \\
5 & 0 & 25 & 6.16258921311118 & $1.4208 \mathrm{e}-014$ & 46 \\
6 & 3 & 24 & 6.23430327124296 & $2.5041 \mathrm{e}-015$ & 36 \\
7 & 4 & 23 & 7.34380858405790 & $8.3404 \mathrm{e}-015$ & 50 \\
8 & 4 & 25 & 7.43120441792176 & $9.8004 \mathrm{e}-015$ & 48 \\
9 & 2 & 25 & 7.63915899496748 & $9.9413 \mathrm{e}-013$ & 38 \\
10 & 5 & 24 & 8.05954250413219 & $2.3375 \mathrm{e}-016$ & \\
\hline
\end{tabular}

*Approximations $\left\{\mu_{k}\right\}_{k=1}^{10}$ to the ten smallest eigenvalues $\left\{\lambda_{k}\right\}_{k=1}^{10}$ of $-\Delta$ on the star-shaped domain with boundary given by $\rho(\theta)=1+0.25 \sin (4 \theta), 0 \leq \theta<2 \pi$. The value $M_{\max }$ indicates the largest value of $M$ used. The number of iterations refers to the number of inner iterations.

\subsection{Polygons}

Next, we move on to piecewise smooth domains, considering polygons. Table 3 lists approximations of the smallest ten eigenvalues on the unit square for the Dirichlet case. These eigenvalues can be computed analytically, and thus we can see that very high accuracy can be obtained in this case, just like for domains with smooth boundaries. Comparable accuracy can be obtained for the case of Neumann boundary conditions on the unit square, as shown in Table 4.

Next, we consider a regular polygon with 128 sides. The eigenvalues for this domain are not known, but in [5], the ten smallest simple eigenvalues were approximated using a three-term Taylor series centered

TABLE 3 Eigenvalues of the unit square, Dirichlet boundary conditions*

\begin{tabular}{rccccc}
\hline$k$ & $m_{0}$ & $M_{\max }$ & $\mu_{k}$ & $\left|\mu_{k}-\lambda_{k}\right| /\left|\lambda_{k}\right|$ & Iteration \\
\hline 1 & 0 & 16 & 19.7392088021787 & 0 & 14 \\
2 & 1 & 18 & 49.3480220054467 & $1.8718 \mathrm{e}-015$ & 29 \\
3 & 2 & 20 & 78.9568352087148 & 0 & 22 \\
4 & 0 & 20 & 98.6960440108938 & $2.8797 \mathrm{e}-015$ & 17 \\
5 & 3 & 20 & 128.3048572141659 & $3.3228 \mathrm{e}-014$ & 29 \\
6 & 1 & 20 & 167.7832748172576 & $7.5186 \mathrm{e}-012$ & 29 \\
7 & 4 & 19 & 177.6528792162691 & $1.8797 \mathrm{e}-011$ & 26 \\
8 & 2 & 17 & 197.3920880213265 & $2.3337 \mathrm{e}-012$ & 41 \\
9 & 5 & 23 & 246.7401100490182 & $8.8288 \mathrm{e}-011$ & 23 \\
10 & 0 & 18 & 256.6097144238706 & $1.7352 \mathrm{e}-011$ & \\
\hline
\end{tabular}

*Approximations $\left\{\mu_{k}\right\}_{k=1}^{10}$ to the ten smallest eigenvalues $\left\{\lambda_{k}\right\}_{k=1}^{10}$ of $-\Delta$ on the unit square for Dirichlet boundary conditions. The value $M_{\max }$ indicates the largest value of $M$ used. 
TABLE 4 Eigenvalues of the unit square, Neumann boundary conditions*

\begin{tabular}{rccccc}
\hline$k$ & $m_{0}$ & $M_{\max }$ & $\mu_{k}$ & $\left|\mu_{k}-\lambda_{k}\right| /\left|\lambda_{k}\right|$ & Iterations \\
\hline 1 & 1 & 15 & 9.8696044010894 & $8.9991 \mathrm{e}-016$ & 28 \\
2 & 2 & 16 & 19.7392088021786 & $2.8797 \mathrm{e}-015$ & 16 \\
3 & 2 & 18 & 39.4784176043572 & $3.9596 \mathrm{e}-015$ & 29 \\
4 & 3 & 18 & 49.3480220054470 & $4.1756 \mathrm{e}-015$ & 31 \\
5 & 4 & 20 & 78.9568352087120 & $3.5097 \mathrm{e}-014$ & 22 \\
6 & 4 & 20 & 88.8264396098096 & $6.0954 \mathrm{e}-014$ & 37 \\
7 & 1 & 17 & 98.6960440063917 & $4.5614 \mathrm{e}-011$ & 25 \\
8 & 5 & 20 & 128.3048572141609 & $5.7594 \mathrm{e}-015$ & 31 \\
9 & 2 & 18 & 157.9136703981950 & $1.2181 \mathrm{e}-010$ & 31 \\
10 & 0 & 21 & 167.7832751306811 & $1.8605 \mathrm{e}-009$ & 34 \\
\hline
\end{tabular}

*Approximations $\left\{\mu_{k}\right\}_{k=1}^{10}$ to the ten smallest nonzero eigenvalues $\left\{\lambda_{k}\right\}_{k=1}^{10}$ of $-\Delta$ on the unit square for Neumann boundary conditions. The value $M_{\max }$ indicates the largest value of $M$ used.

at the eigenvalues of the circle,

$$
\lambda_{n}^{(N)}=\lambda_{n}\left(1+\frac{2}{3} \frac{\pi^{2}}{N^{2}}+\frac{1}{6} \frac{\pi^{3}}{N^{3}}\right)
$$

where $N$ is the number of sides in the polygon, and $\lambda_{n}$ is the $n$th simple eigenvalue for the circle. Obviously, this approximation is most suitable for regular polygons with many sides.

The approximations listed in Table 5 were obtained by considering only the case where $M=0$ and $m_{0}=0$. The discretization of the polygon used to construct $A(r, 0,0)$ consisted of 32 points per side, and experimentation with other discretizations showed quadratic convergence in the number of points. Because the boundary of the polygon is well-approximated by a circle, zeros of Bessel functions provide very

TABLE 5 Eigenvalues of a 128-sided regular polygon*

\begin{tabular}{|c|c|c|c|c|c|}
\hline$k$ & $m_{0}$ & $\mu_{k}$ & $\lambda_{k}$ & $\left|\mu_{k}-\lambda_{k}\right| /\left|\lambda_{k}\right|$ & Iterations \\
\hline 1 & 0 & 5.78551 & 5.78552 & $2.2801 \mathrm{e}-006$ & 2 \\
\hline 2 & 0 & 30.48349 & 30.48357 & $2.6001 \mathrm{e}-006$ & 2 \\
\hline 3 & 0 & 74.91706 & 74.91726 & $2.6745 \mathrm{e}-006$ & 2 \\
\hline 4 & 0 & 139.09608 & 139.09646 & $2.7135 \mathrm{e}-006$ & 2 \\
\hline 5 & 0 & 223.02177 & 223.02237 & $2.6975 \mathrm{e}-006$ & 2 \\
\hline 6 & 0 & 326.69441 & 326.69528 & $2.6759 \mathrm{e}-006$ & 2 \\
\hline 7 & 0 & 450.11409 & 450.11529 & $2.6638 \mathrm{e}-006$ & 2 \\
\hline 8 & 0 & 593.28086 & 593.28245 & $2.6746 \mathrm{e}-006$ & 2 \\
\hline 9 & 0 & 756.19474 & 756.19675 & $2.6567 \mathrm{e}-006$ & 2 \\
\hline 10 & 0 & 938.85573 & 938.85822 & $2.6481 \mathrm{e}-006$ & 2 \\
\hline
\end{tabular}

*Approximations $\left\{\mu_{k}\right\}_{k=1}^{10}$ to the ten smallest simple eigenvalues of $-\Delta$ on the 128-sided regular polygon. These approximations are compared with those computed in [5], denoted by $\lambda_{k}$, $k=1,2, \ldots, 10$. 
accurate initial iterates, thus making iteration beyond $M=0$ unnecessary. Nonsimple eigenvalues can be obtained by considering other values of $m_{0}$, whereas the Taylor series approach is designed specifically for computing simple eigenvalues.

\subsection{Detecting Multiple and Clustered Eigenvalues}

Multiple eigenvalues and closely clustered eigenvalues can be detected using the smallest singular values of $A\left(r, M, m_{0}\right)$. Specifically, if $r^{2}$ is an eigenvalue of multiplicity $k$, then we can expect that the $k$ smallest singular values of $A\left(r, M, m_{0}\right)$ should be nearly zero if $M$ is sufficiently large. Furthermore, if the $(k+1)$ st singular value is small, then this suggests that another eigenvalue is nearby.

To illustrate this, we consider the case of the rectangle $[0,1] \times[0,1+\epsilon]$, which, for Dirichlet boundary conditions, has eigenvalues $\pi^{2}\left(m^{2}+\right.$ $\left.(n /(1+\epsilon))^{2}\right)$ for positive integers $m$ and $n$. This leads to several double eigenvalues when $\epsilon=0$, and clustered eigenvalues for small nonzero values of $\epsilon$. In Table 6, we consider these cases: (1) $m=1$ and $n=2$, and (2) $m=2$ and $n=1$. For $\epsilon=0$, these cases result in a double eigenvalue of $5 \pi^{2}$, whereas for $\epsilon=10^{-6}$, they yield two distinct eigenvalues that differ by $5.92 \times 10^{-5}$. Our algorithm will only find one of them, but for the computed eigenvalue $r_{1}^{2}$, the second smallest singular value of $A\left(r_{1}, M, m_{0}\right)$ is quite small, indicating that there exists a linear combination of functions of the form $J_{m}\left(r_{1} \rho(\theta)\right) e^{i m \theta}$ that, while orthogonal to the computed eigenfunction, nearly satisfies the boundary conditions. It is therefore reasonable to conclude that there is a second eigenvalue $r_{2}^{2}$ nearby, which can be found by scanning the graph of $\sigma_{1}\left(A\left(r, M, m_{0}\right)\right)$ near $r_{1}$.

We can not only use the second smallest singular value to detect the presence of another nearby eigenvalue but also determine its approximate location. In Figure 4, we see that after finding either of the eigenvalues shown in the figure, we can approximate the other one by computing the horizontal intercept of a secant line for the graph of the second smallest

TABLE 6 Detecting clustered Eigenvalues*

\begin{tabular}{lcccc}
\hline$\epsilon$ & $\lambda$ & $\operatorname{mult}(\lambda)$ & $\sigma_{1}\left(A\left(\sqrt{\lambda}, M, m_{0}\right)\right)$ & $\sigma_{2}\left(A\left(\sqrt{\lambda}, M, m_{0}\right)\right)$ \\
\hline 0 & 49.34802200544679 & 2 & 0 & 0 \\
$1 \mathrm{e}-6$ & 49.34794304872997 & 1 & 0 & $3.8616 \mathrm{e}-006$ \\
\hline
\end{tabular}

*Smallest two singular values $\sigma_{1}$ and $\sigma_{2}$ of the matrix $A\left(\sqrt{\lambda}, M, m_{0}\right)$ for the domain $[0,1] \times[0,1+\epsilon]$, with $m_{0}=0$ and $M=20$. For $\epsilon=0$, the Laplacian has a double eigenvalue at 49.34802200544679 , and for $\epsilon=10^{-6}$, there are two simple eigenvalues at 49.34794304873002 and 49.34800226626760 . 


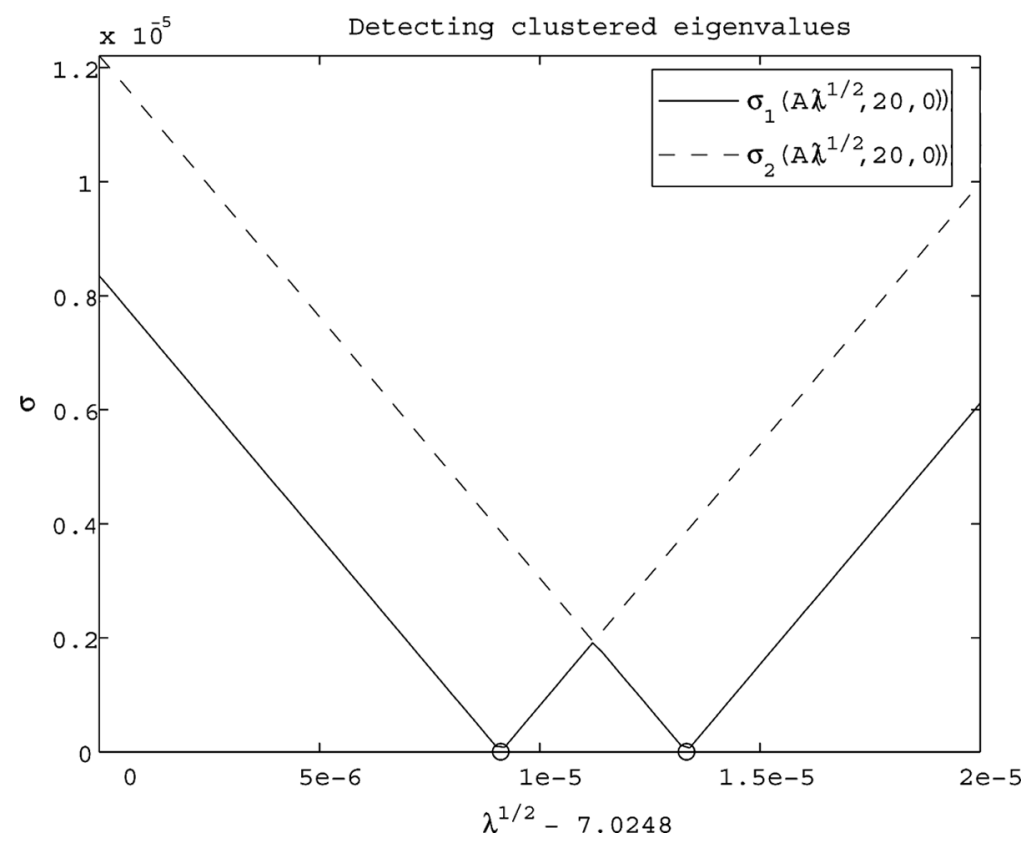

FIGURE 4 Smallest two singular values $\sigma_{1}$ (solid curve) and $\sigma_{2}$ (dashed curve) of the matrix $A\left(\sqrt{\lambda}, M, m_{0}\right)$ for the domain $[0,1] \times[0,1+\epsilon]$, with $m_{0}=0, M=20$, and $\epsilon=10^{-6}$. The exact eigenvalues are located at the circles on the horizontal axis.

singular value near the first eigenvalue. It should be noted that in [4], similar behavior of the second smallest singular value was observed, and this behavior is also exploited in the scaling method introduced by Vergini and Saraceno in [12] and improved by Barnett in [13].

\subsection{Domains with Both Singular and Regular Corners}

For more challenging test cases, we consider domains that have both regular and singular corners, where a regular corner is a corner with interior angle $\pi / \alpha$ for an integer $\alpha$ and a singular corner is a corner that is not regular. First, we consider an $L$-shaped domain, defined by

$$
\rho(\theta)=\frac{1}{\max (|\sin \theta|,|\cos \theta|)}, \quad 0 \leq \theta<\frac{3 \pi}{2},
$$

for which eigenvalues were computed in [4] using a modification of the MPS introduced in [3]. This domain has a singular corner of angle $3 \pi / 2$. For this reason, instead of using the eigenfunctions for the free space operator, we use the eigenfunctions for the operator defined on an 
TABLE 7 Eigenvalues of an $L$-shaped domain*

\begin{tabular}{ccccc}
\hline$k$ & $\mu_{k}$ & $\lambda_{k}$ & $\left|\lambda_{k}-\mu_{k}\right| /\left|\lambda_{k}\right|$ & Iterations \\
\hline 1 & 9.63971927923382 & 9.63972384464540 & $4.7360 \mathrm{e}-007$ & 10 \\
2 & 15.19727667220087 & 15.19725192576365 & $1.6283 \mathrm{e}-006$ & 11 \\
3 & 19.73920878892437 & 19.73920880208238 & $6.6659 \mathrm{e}-010$ & 8 \\
\hline
\end{tabular}

*Approximations $\left\{\mu_{k}\right\}_{k=1}^{3}$ to the three smallest eigenvalues of $-\Delta$ on an $L$-shaped domain using Fourier-Bessel functions of order $2 \mathrm{~m} / 3$ for each positive integer $m$, centered at the singular corner. These approximations are compared with those computed in [4], denoted by $\lambda_{k}, k=1,2,3$.

unbounded wedge of interior angle $3 \pi / 2$, which are the Fourier-Bessel functions

$$
u_{r, m}(\rho, \theta)=J_{2 m / 3}(r \rho) \sin \frac{2 m \theta}{3}, \quad m=0,1, \ldots
$$

which will vanish on the sides of the boundary that define the singular corner.

The algorithm proceeds as described in the previous section, except that the series (2.5) is a linear combination of these eigenfunctions, and the entries of the matrix $A\left(r, M, m_{0}\right)$ are obtained by taking inner products with functions of the form $\sin (2 m \theta / 3)$ rather than $e^{i m \theta}$, in view of the discussion at the end of Section 3.1. Table 7 contains the resulting approximations $\left\{\lambda_{k}\right\}_{k=1}^{3}$ of the smallest three eigenvalues, compared with the approximations $\left\{\mu_{k}\right\}_{k=1}^{3}$ reported in [4]. It should be noted that both sets of results were obtained using MATLAB implementations of the respective algorithms, run on a Toshiba Satellite A75-211 Notebook with a Pentium 4 processor. Our algorithm computed these three eigenvalues in 1.11 seconds, whereas the modified MPS algorithm (see [14]) did so in 2.31 seconds. In both cases, 72 points were used in the discretization of the boundary, and the modified MPS algorithm also used 72 randomly chosen interior points.

Next, we use the original version of the algorithm, with free space eigenfunctions and an expansion centered at an interior point of the domain instead of at the singular corner. As shown in Table 8, we obtain only a few correct digits for the first two eigenvalues, while still achieving high accuracy for the third, which coincides with an eigenvalue of the unit square.

Finally, we try our algorithm on one of the Gordon-Webb-Wolpert (GWW) drums described in [15], the GWW1 drum shown in Figure 5. We again use free space eigenfunctions centered in the interior, and a discretization of Chebyshev points on each side, so that points will be clustered near each corner. The results are shown in Table 9. As with the $L$-shaped domain, we obtain at most a few digits. However, it is clear that our algorithm can at least be used to obtain an initial approximation for 


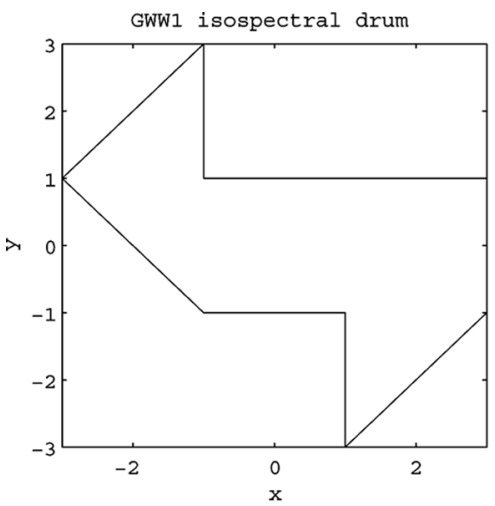

(a)

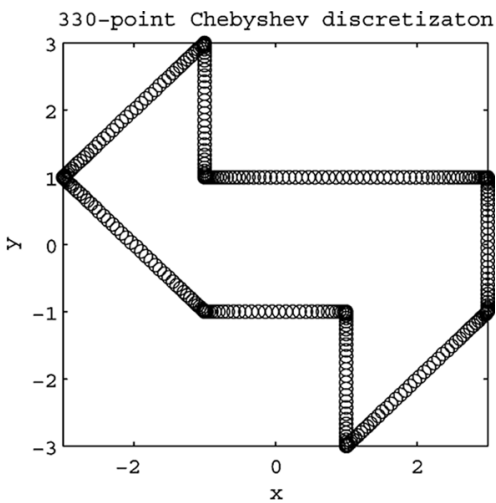

(b)

FIGURE 5 Left plot: the GWW1 drum. Right plot: a 330-point discretization of the drum, using Chebyshev points on each side.

a method such as the MPS, and future work will focus on improving our algorithm for such domains.

\subsection{An Inverse Problem}

We now consider an inverse spectral problem, in which $n$ eigenvalues $\lambda_{1}, \ldots, \lambda_{n}$ of $-\Delta$ are specified, and our goal is to find a domain $\Omega$ such that $-\Delta$ has eigenfunctions that vanish on $\partial \Omega$ with corresponding eigenvalues equal to the prescribed values. The solution of this problem is not unique, as discussed in [15], but we hope to obtain a domain whose boundary is close to our initial guess. To that end, we define the matrix $A\left(\rho, r, M, m_{0}\right)$ in the same way as we defined $A\left(r, M, m_{0}\right)$ in the previous section, except that in this case, the function $\rho(\theta)$ that defines the boundary is allowed to vary. Then, we solve the problem of finding $\rho \in W_{n}$ that minimizes

$$
f(\rho)=\sum_{i=1}^{n} \sigma_{1}\left(A\left(\rho, \sqrt{\lambda_{i}}, M^{(i)}, m_{0}^{(i)}\right)\right),
$$

TABLE 8 Eigenvalues of an $L$-shaped domain, ignoring singular corners*

\begin{tabular}{|c|c|c|c|c|c|}
\hline$k$ & $m_{0}$ & $\mu_{k}$ & $\lambda_{k}$ & $\left|\lambda_{k}-\mu_{k}\right| /\left|\lambda_{k}\right|$ & Iterations \\
\hline 1 & 0 & 9.72384639102523 & 9.63972384464540 & 0.00873 & 70 \\
\hline 2 & 2 & 15.16260753106696 & 15.19725192576365 & 0.00228 & 72 \\
\hline 3 & 2 & 19.73920880217876 & 19.73920880208238 & $1.2373 \mathrm{e}-011$ & 30 \\
\hline
\end{tabular}

*Approximations $\left\{\mu_{k}\right\}_{k=1}^{3}$ to the three smallest eigenvalues of $-\Delta$ on an $L$-shaped domain using free space eigenfunctions centered in the interior, with $M=40$. These approximations are compared with those computed in [4], denoted by $\lambda_{k}, k=1,2,3$. 
TABLE 9 Eigenvalues of a GWW drum, ignoring singular corners*

\begin{tabular}{cccccc}
\hline$k$ & $m_{0}$ & $\mu_{k}$ & $\lambda_{k}$ & $\left|\lambda_{k}-\mu_{k}\right| /\left|\lambda_{k}\right|$ & Iterations \\
\hline 1 & 0 & 2.55996542353383 & 2.537943999798 & 0.00868 & 78 \\
2 & 1 & 3.67093788732147 & 3.655509713520 & 0.00422 & 78 \\
3 & 2 & 5.16128092767208 & 5.175559356220 & 0.00458 & 78 \\
\hline
\end{tabular}

*Approximations $\left\{\mu_{k}\right\}_{k=1}^{3}$ to the three smallest eigenvalues of $-\Delta$ on the GWW1 drum, with $M=40$. These approximations are compared with those computed in [4], denoted by $\lambda_{k}, k=1,2,3$.

where $W_{n}$ is a subspace of $C_{p}([0,2 \pi])$, and $M^{(i)}$ and $m_{0}^{(i)}$ determine the size and center of the eigenfunction expansion corresponding with $\lambda_{i}$.

We illustrate the results of this approach with a simple example. We use eight prescribed eigenvalues of the domain

$$
\rho(\theta)=1+0.05(\cos \theta+\sin 2 \theta+\cos 3 \theta)
$$

computed using the modified MPS, as it is ill-advised to test a method for solving an inverse problem on data obtained using the same method for the forward problem. We then define

$$
W_{8}=\operatorname{span}\{1, \cos \theta, \cos 2 \theta, \cos 3 \theta, \sin \theta, \sin 2 \theta, \sin 3 \theta\},
$$

and choose $M^{(i)}=8$ and $m_{0}^{(i)}=0$ for $i=1, \ldots, 8$. Our initial guess is a perturbation of the exact solution,

$$
\rho_{0}(\theta)=1.01+0.04(\cos \theta+\sin 2 \theta+\cos 3 \theta) .
$$

We solve the problem (4.1) using MATLAB's fminsearch function with initial guess $\rho_{0}(\theta)$ and a stopping criterion that the value of the objective function should be less than $10^{-10}$. The output is a function $\rho_{1}(\theta)$ that satisfies $\left\|\rho_{1}-\rho\right\| \approx 1.1 \times 10^{-3}\|\rho\|$, whereas $\left\|\rho_{0}-\rho\right\| \approx 1.2 \times 10^{-2}\|\rho\|$. The domains defined by $\rho, \rho_{0}$ and $\rho_{1}$ are shown in Figure 6 .

Figure 7 shows the value of the objective function from (4.1) after each iteration performed by fminsearch. Note that there are periods of relatively steep drops in the function value, between periods of relatively little change. This suggests that the algorithm is finding multidimensional "wells" as it searches $W_{n}$, corresponding with the one-dimensional wells observed in the solution of the forward problem. Future work will involve attempting to develop an optimization method that can find these wells as quickly as possible, even if minimizing over a higher-dimensional function space. 


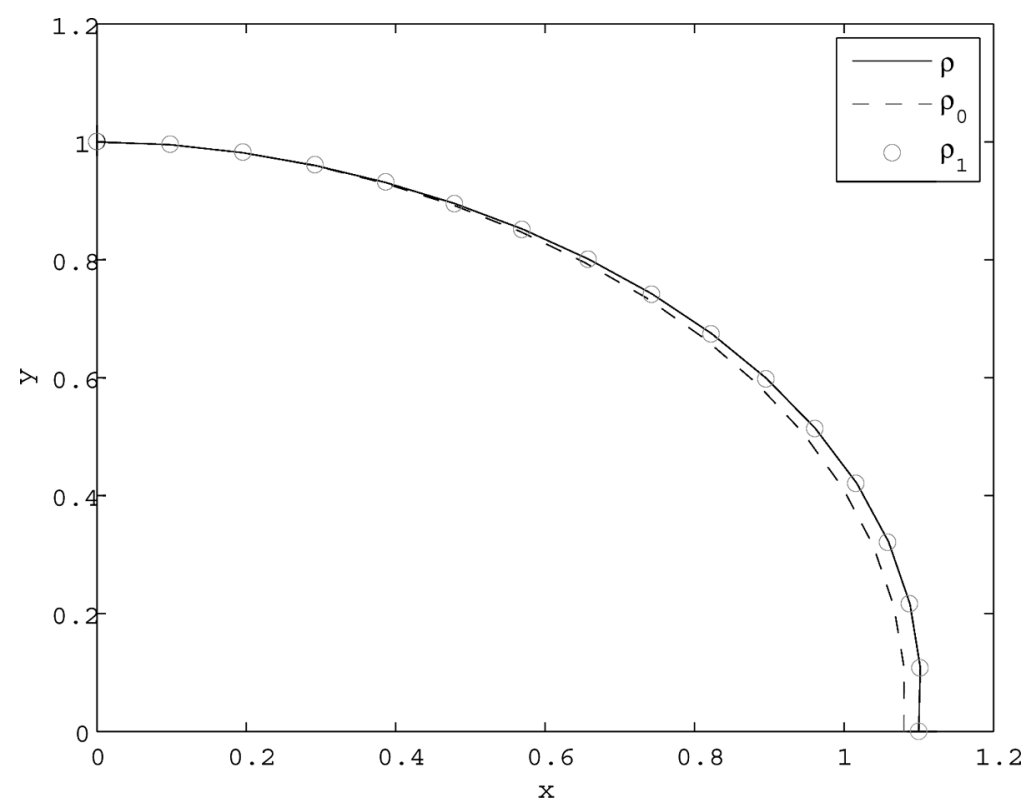

FIGURE 6 Results of solving the inverse eigenvalue problem described in Section 4.5. The solid curve is the exact solution $\rho(\theta)$ defined in (4.2), the dashed curve is the initial guess $\rho_{0}(\theta)$, and the open circles describe the computed solution $\rho_{1}(\theta)$.

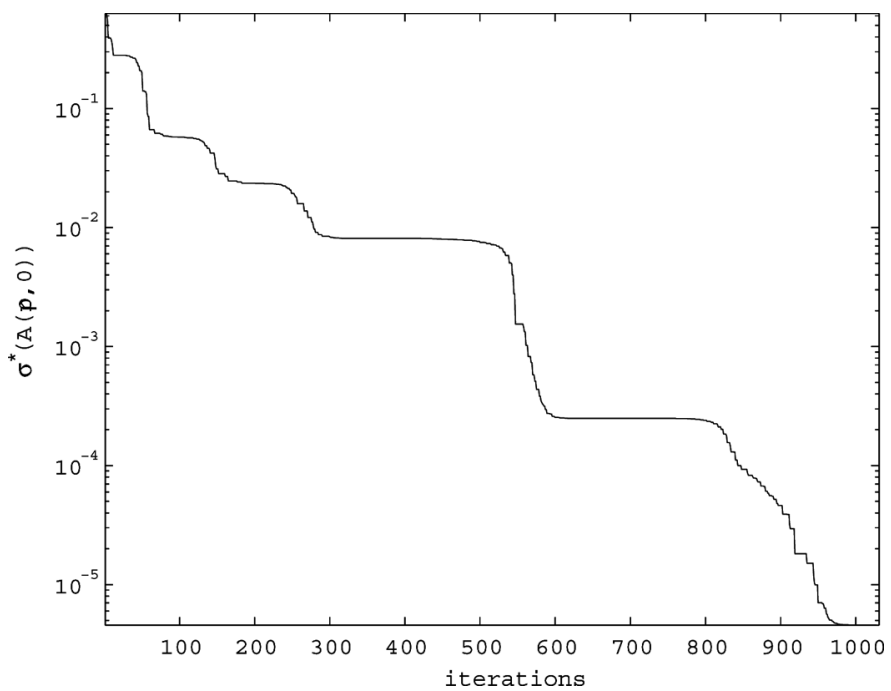

FIGURE 7 The value of the objective function $f(\rho)$, defined in (4.1), after each iteration performed by fminsearch. 


\subsection{Comparison with the Method of Particular Solutions}

The method of particular solutions (MPS), introduced by Fox, Henrici, and Moler in [3] and revived by Betcke and Trefethen in [4], can be used to compute eigenvalues of the Laplacian by solving an equation such as (2.8). Our approach is similar to the MPS in that both algorithms locate an approximate eigenvalue $\lambda$ by minimizing a function $f(\lambda)$ whose value is the smallest singular value of some matrix $A(\lambda)$. In both algorithms, the columns of $A(\lambda)$ correspond with select eigenfunctions of the Laplacian on some unbounded domain, a linear combination of which is taken in (2.8). In [13], Barnett presents another modification of the MPS, in which robustness is achieved by solving a generalized eigenvalue problem in order to minimize the ratio of a norm of an approximate eigenfunction on the boundary to its norm on the interior.

The main difference between our approach and any of these three variations of the MPS is that rather than minimizing, in some sense, a linear combination of the restriction of the free space eigenfunctions to the boundary, we minimize its projection into some subspace, such as the columns of $Q\left(\epsilon, M, m_{0}\right)$ defined in Section 3.1. To explain the benefit of this tactic, we consider where MPS, and its variants, may be least efficient.

Let $\widetilde{A}(r)$ be an $m \times n$ matrix whose columns consist of the values of free space eigenfunctions at select points on the boundary. The MPS, and its modification from [4], locate an eigenvalue by computing the smallest singular value of such a matrix, whereas the modified MPS in [13] computes the smallest generalized eigenvalue with respect to a matrix $\widetilde{B}(r)$ consisting of values of the same eigenfunctions at interior points. If the number of columns, $n$, is very small, then this smallest singular value or generalized singular value is not near zero for any value of $r$, as illustrated in Figure 8. It follows that it is not practical to employ a secant-line approach such as that described in Section 3.2, which can also be employed by the MPS when $n$ is sufficiently large. Instead, an alternative approach must be used such as repeatedly fitting the objective function with a quadratic polynomial and minimizing, which can lead to a much greater number of iterations than in cases for which a secant-line approach is feasible. Furthermore, note that in Figure 8, the minimia are still not very close to the exact eigenvalues.

Our goal is to use projection to transform the previously described problem of minimizing the smallest singular value into, essentially, the problem of finding a zero of a function that is piecewise linear near its roots, for which a secant-line approach can be employed to more rapidly find a solution, even if the number of columns is small. To obtain some guidance on the question of choosing the subspace into which the columns of $\widetilde{A}(r)$ should be projected, suppose that an approximate eigenfunction 


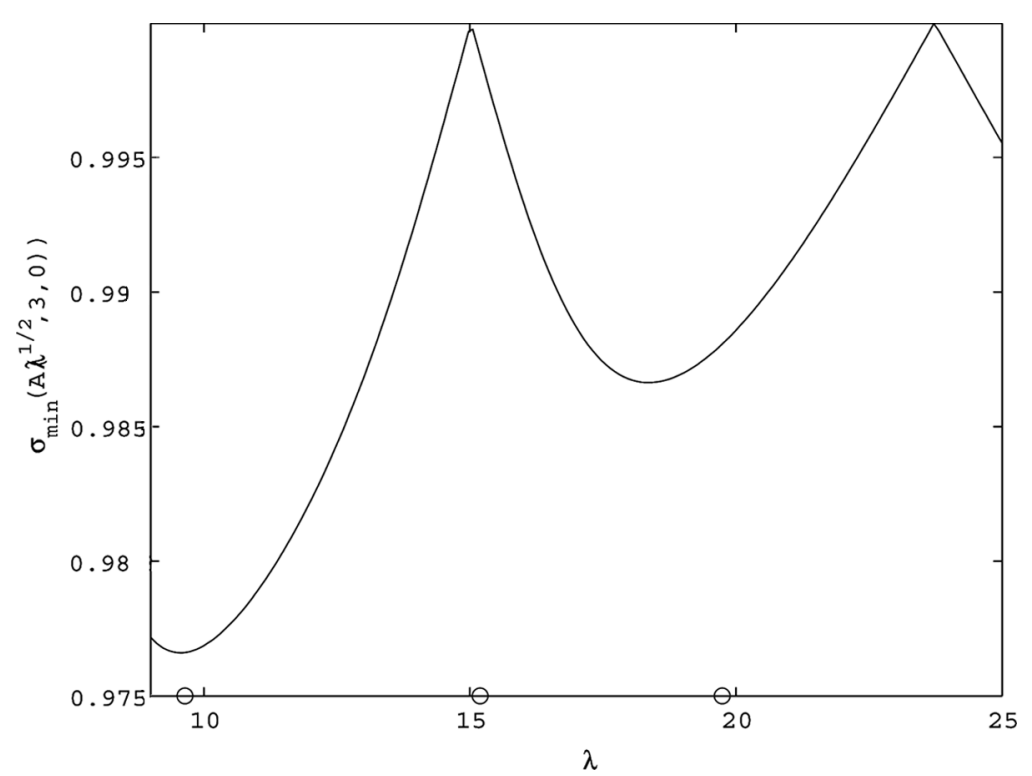

FIGURE 8 The smallest singular value of $A\left(r, M, m_{0}\right)$ for $M=3, m_{0}=0$, where $r=\sqrt{\lambda}$, for the $L$-shaped domain. The circles represent the exact values of the smallest three eigenvalues of this domain.

$u(x, y)$ with corresponding approximate eigenvalue $\lambda$ is represented as a finite linear combination of eigenfunctions for an unbounded wedge,

$$
u(x, y)=\sum_{m=1}^{M} \alpha_{m} u_{m}(x, y), \quad u_{m}(x, y)=J_{2 m / 3}(2 \sqrt{\lambda}|x| / 3) \sin \left(2 m \theta_{x} / 3\right)
$$

where $M$ is chosen sufficiently large to obtain high accuracy. Then, choose $\widetilde{M} \ll M$ and consider the matrix $A(r, \widetilde{M}, 0)$ as defined in Section 3.1, except that only positive indices are used. That is, the $m$ th column of $A(r, \widetilde{M}, 0)$ consists of the values of $u_{m}(x, y)$ at the boundary points chosen for the discretization.

Next, we let $B(r, \widetilde{M}, 0)$ be an orthogonal matrix whose columns are also orthogonal to the discretizations of $\left\{u_{m}(x, y)\right\}_{m>\widetilde{M}}$ on the boundary. In other words, if we write

$$
A(r, M, 0)=\left[\begin{array}{cc}
A_{1} & A_{2} \\
\widetilde{M} & M-\tilde{M}
\end{array}\right]
$$

where the columns of $A(r, M, 0)$ are ordered by increasing Bessel function index, so that $A_{1}=A(r, \widetilde{M}, 0)$, then $B(r, \widetilde{M}, 0)^{*} A_{2}=0$. Furthermore, $C(r, \widetilde{M}, 0) \vec{\alpha}=B(r, \widetilde{M}, 0)^{*} A(r, \widetilde{M}, 0) \vec{\alpha} \approx 0$, where $\vec{\alpha}=\left[\alpha_{1} \cdots \alpha_{\widetilde{M}}\right]$, even if $\widetilde{M}$ 
is chosen to be so small that the matrix $A(r, \widetilde{M}, 0)$, with $\widetilde{M}$ columns, is not nearly singular.

Figure 9 displays the graph of $C(r, \widetilde{M}, 0)$ as a function of $r$. Note that this function, numerically, has zeros near the locations of the exact eigenvalues, whereas $A(r, \widetilde{M}, 0)$, shown in Figure 8 , does not. It follows that approximate eigenvalues can be obtained much more rapidly from $C(r, \tilde{M}, 0)$, as a secant-line approach can be employed, significantly reducing the number of evaluations of the objective function. The reduction in the number of evaluations, however, is only worthwhile if $C\left(r, \widetilde{M}, m_{0}\right)$ can be constructed efficiently, and this requires an approximation of the orthogonal complement represented by $B\left(r, \widetilde{M}, m_{0}\right)$.

If the boundary is sufficiently smooth, then a crude but useful approximation is obtained by simply performing the Fourier transform of the columns of $A\left(r, \widetilde{M}, m_{0}\right)$ and filtering out all but the lowest $n$ frequencies. As the results in the previous section indicate, even for difficult domains such as the isospectral drum, accurate initial estimates of eigenvalues can be obtained by using an approximation of $B\left(r, \widetilde{M}, m_{0}\right)$ that is independent of $r$, such as $Q\left(\epsilon, \widetilde{M}, m_{0}\right)$ from Section 3.1.

We have seen in this section that these differences can lead to greater computational efficiency for our approach, especially for the task of obtaining accurate initial estimates of eigenvalues. For domains with both regular and singular corners, our algorithm can be used in conjunction

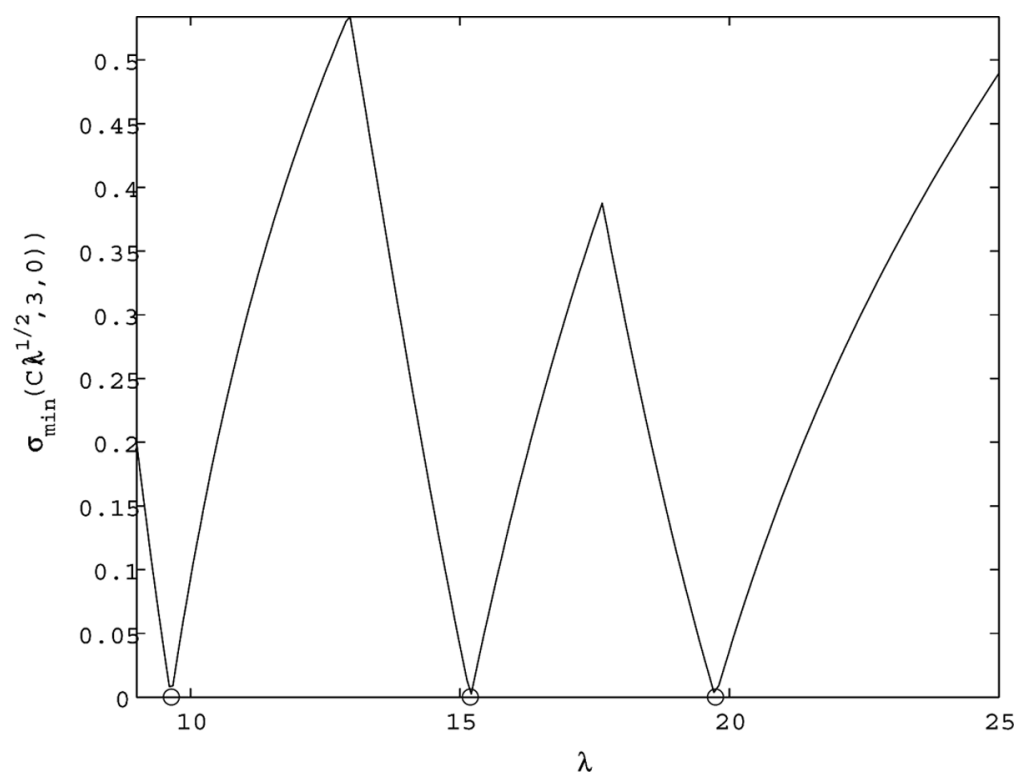

FIGURE 9 The smallest singular value of $C\left(r, M, m_{0}\right)$ for $M=3, m_{0}=0$, where $r=\sqrt{\lambda}$, for the $L$-shaped domain. The circles represent the exact values of the smallest three eigenvalues of this domain. 
with MPS to compute eigenvalues with high accuracy and greater efficiency than with either method used alone.

\section{CONCLUSIONS}

An iterative method for the numerical computation of eigenvalues has been proposed that relies on calculations performed on small matrices and that allows for the independent targeting of individual eigenvalues. Furthermore, the method does not require the evaluation of functions at points in the interior of the domain, and it does not require exhaustive scanning of the positive real axis. Numerical experiments and comparisons corroborate these claims. It is our hope that the approach described in this work will eventually lead to effective methods for solving a variety of PDEs on general 2-D and 3-D domains, as well as insight concerning the eigensystem of the Laplacian and related operators on such domains.

\section{REFERENCES}

1. J. Descloux and M. Tolley (1983). An accurate algorithm for computing the eigenvalues of a polygonal membrane. Comput. Methods Appl. Mech. Eng. 39:37-53.

2. H. Wu, D. Sprung, and J. Martorell (1995). Numerical investigations of isospectral cavities built from triangles. Phys. Rev. E. 51:703-708.

3. L. Fox, P. Henrici, and C. Moler (1967). Approximations and bounds for eigenvalues of elliptic operators. SIAM J. Numer. Anal. 4:89-102.

4. T. Betcke and L. N. Trefethen (2005). Reviving the method of particular solutions. SIAM Rev. 47:469-491.

5. G. Strang and P. Grinfeld (2004). The Laplacian eigenvalues of a polygon. Computers Math. Appl. 48:1121-1133.

6. J. V. Lambers (2005). Krylov subspace spectral methods for variable-coefficient initial-boundary value problems. Elec. Trans. Numer. Anal. 20:212-234.

7. R. Courant and D. Hilbert (1989). Methods of Mathematical Physics. Wiley Interscience, New York.

8. M. Abramowitz and I. A. Stegun (1970). Handbook of Mathematical Functions. Dover, New York.

9. G. H. Golub and C. F. van Loan (1996). Matrix Computations. The Johns Hopkins University Press, Baltimore, MD.

10. J. Baglama and L. Reichel (2006). Restarted block Lanczos bidiagonalization methods. Numer. Algorithm. 43:251-272.

11. E. Weisstein. Bessel Function. From MathWorld-A Wolfram Web Resource. Available at: http:// mathworld.wolfram.com/BesselFunction.html.

12. E. Vergini and M. Saraceno (1995). Calculation by scaling of highly excited states of billiards. Phys. Rev. E. 52:2204.

13. A. H. Barnett (2000). Ph.D. thesis, Harvard University.

14. T. Betcke and L. N. Trefethen (2003). Ldrum.m-eigenvalues of Laplacian on L-shaped region (MATLAB implementation). Available at: http://web.comlab.ox.ac.uk/oucl/work/nick.trefethen/ Ldrum.m.

15. C. Gordon, G. Webb, and S. Wolpert (1992). Isospectral plane domains and surfaces via Riemannian orbifolds. Invent. Math. 110:1-22. 